\title{
Toxic effects of copper on liver and cholinesterase of Clarias gariepinus
}

\begin{abstract}
The release of pollutants, especially heavy metals, into the aquatic environment is known to have detrimental effects on such an environment and on living organisms including humans when those pollutants are allowed to enter the food chain. The aim of this study is to analyse the damage to Clarias gariepinus' liver caused by exposure to different concentrations of copper. In the present study, samples of C. gariepinus were exposed to sub-lethal copper sulphate (CuSO4) concentrations (from 0.2 to $20.0 \mathrm{mg} / \mathrm{L}$ ) for $96 \mathrm{~h}$. Physiological and behavioural alterations were observed with respect to their swimming pattern, mucus secretion and skin colour. Mortality was also observed at high concentrations of copper. Histopathological alterations of the liver were analysed under light, transmission and scanning electron microscopies. The liver of the untreated group showed normal tissue structures, while histopathological abnormalities were observed in the treated fish under light and electron microscopes with increased copper concentrations. Histopathological abnormalities include necrosis, melanomacrophage, hepatic fibrosis and congested blood vessels. In addition, the enzyme activity of liver cholinesterase (ChE) was also found to be affected by copper sulphate, as $100 \%$ of cholinesterase activity was inhibited at $20.0 \mathrm{mg} / \mathrm{L}$. Thus, liver enzyme activity and histopathological changes are proven to be alternative sources for biomarkers of metal toxicity.
\end{abstract}

Keyword: Copper contamination; Cholinesterase; Clarias gariepinus; Biomarker; Acute toxicity; Histopathology effect 